\title{
Marktplatzbummel als Tour im Stände-Dickicht
}

Fragen zu und Argumente für diese provozierende Kapitalüberschrift:

- Warum braucht man einen Marktplatz zur Verdeutlichung der GRV? Weil viele Finanzberater, Anlageberater, Vermögensberater nicht die Prüfkriterien als AV-Berater erfüllen sind, da sie die GRV-Spezifika zu wenig kennen und sie dafür nur eingeschränktes Interesse haben?

- Warum braucht man einen bAV-Marktplatz zur Popularitätsförderung der bAV? Weil bAV auf Großunternehmen mit Tarifbindungen weitgehend beschränkt ist und auf den Mittelstand wenig ausstrahlt!

- Warum braucht man den Riester-Marktplatz mit Riester-Sachverständigen? Weil Riester-Produkte zu sehr aus Produktgebersicht beraten werden und die Mosaik-Bedarfssituation zu wenig berücksichtigt wird!

- Wie kommt man zur ganzheitlichen AV-Beratung ohne Tunnelblick? Welche Wege sind Erfolg versprechend? Honorarberater? Berater in Verbünden, die zunächst unabhängige AV-Beratung auf ihre Fahnen geschrieben haben, bevor sie an Verkauf denken?

Das deutsche AV-System ist im Gegensatz zu der Mehrzahl der leistungsfähigeren EU-AV-Systeme nicht einfach für den Bürger durchschaubar, geschweige denn selbsterklärend. Gegenbeispiele sind die Systeme der Schweiz und der Niederlande: Man gibt an, wie viel man bereit ist (in der Schweiz ab einem Jahreseinkommen von umgerechnet von 20.000 Franken aufwärts), in der bAV anzulegen bzw. bei Arbeitnehmern wird es obligatorisch einbehalten. Dementsprechend einfach ist die Verwaltung.

Fazit: Die Bürger brauchen ein AV-System, in dem sie ihren AV-Bedarf mit für sie selbst verständlichen Worten ,auf dem Bierdeckel“ artikulieren können - ein neues System! 